\title{
Coronary sinus reentrant tachycardia after atrial fibrillation ablation: From bad to worse
}

\author{
Pietro Turco \\ Electrofisiology Laboratory, Multimedica Hospital, Milano, Italia \\ Email: pietro.turco@tin.it
}

Received 29 October 2013; revised 8 December 2013; accepted 17 December 2013

Copyright (c) 2014 Pietro Turco. This is an open access article distributed under the Creative Commons Attribution License, which permits unrestricted use, distribution, and reproduction in any medium, provided the original work is properly cited. In accordance of the Creative Commons Attribution License all Copyrights (C) 2014 are reserved for SCIRP and the owner of the intellectual property Pietro Turco. All Copyright (c) 2014 are guarded by law and by SCIRP as a guardian.

\section{ABSTRACT}

Herein we present a case of atrial tachycardia as a sequel of AF ablations. A 42-year-old man was admitted to our department because of a very symptomatic tachycardia. The patient, because of paroxysmal AF and typical atrial flutter, had been already submitted (three times) to ablation procedures in both left (pulmonary vein insulation) and right atria (cavo-tricuspidal isthmus). During the electrophysiological study, a huge and very fast atrial tachycardia was induced: 230 ms cycle length, $1 / 1$ atrio-ventricular conduction with the ventricular rate of $260 \mathrm{bpm}$, complete left bundle branch block, and clinically recognized by the patient. Four minutes later, a 2/1 AV conduction without branch block permitted mapping and ablation. A high-density mapping around isthmus and coronary sinus (CS) was performed. The analysis of the chronological activation clearly showed a circuit propagation around the CS ostium with a very slow conduction in the anterior zone enlightened by the tight color progression, and counterclockwise activation of the right atrium lateral wall. In anterior zone of CS ostium diastolic fragmented electrograms were detected. After preventing his position localization, radiofrequency delivering $(35 \mathrm{~W})$ was effective

\footnotetext{
${ }^{*}$ Core Tip. The increased number of transcatheter ablations in the atria for treatment of atrial fibrillation is contributing to an increased incidence of organized macro reentrant tachycardia.

Herein we present a case of atrial tachycardia as a sequel of atrial fibrillation ablations. During EP study a huge very fast, 260 bpm, atrial tachycardia was induced. A high-density mapping (Carto) indicated a reentry circuit around CS. In anterior edge of CS diastolic fragmented electrograms were localized. Radiofrequency delivering was effective to interrupt the arrhythmia. Energy delivering was continued to anchor the lesion to the inferior vena cava. In conclusion, extensive liner lesions in right and left atria and normal anatomical barriers (tricuspidal annulus, Eustachian ridge and crista terminalis, scars) could contribute to the onset of conduction corridors (man-made maze) that could sustain more aggressive arrhythmias.
}

to interrupt the arrhythmia in 3 seconds. Energy delivering was continued to anchor the lesion to the inferior vena cava. Confirmation of successful ablation was determined by unsuccessful attempts at reinduction of the arrhythmia, in basal state and during infusion of isoproterenol.

\section{KEYWORDS}

Atrial Flutter; Atrial Fibrillation; Transcatheter Ablation; Three-Dimensional Mapping

\section{INTRODUCTION}

The increased number of transcatheter ablations in the atria for treatment of atrial fibrillation (AF) contributes to an increased incidence of organized macro reentrant tachycardia [1-4].

Herein we present a case of atrial tachycardia as a sequel of AF ablations.

\section{CASE REPORT}

A 42-year-old man was admitted to our department because of a very symptomatic tachycardia. The patient, because of paroxysmal AF and typical atrial flutter, had been already submitted (three times) to ablation procedures in both left (pulmonary vein insulation) and right atria (cavo-tricuspidal isthmus).

In spite of anti-arrhythmic therapy, the patient, four days before his admission, experienced a new very symptomatic (sweating, hypotension and dizziness) tachycardia for which was submitted to ablation procedure again.

At the procedure the patient, on pharmacological washout, presented a stable sinus rhythm.

By coronary sinus (CS) stimulation, with extra stimulus technique, a huge very fast atrial tachycardia was induced: 230 ms cycle length, 1/1 atrio-ventricular con- 
duction with ventricular rate of $260 \mathrm{bpm}$, complete left bundle branch block and clinically recognized by the patient (Figure 1). Four minutes later a 2/1 AV conduction without branch block permitted mapping and ablation (Figure 2).

A 7.5 F $3.5 \mathrm{~mm}$ irrigated-tip roving catheter (Navistar Thermocool, Diamond Bar, CA, USA) inserted by right femoral vein for electroanatomical mapping and ablation was used.

Electroanatomical mapping (CARTO 3, Diamond Bar, CA, USA) commenced in the right atrium because of the CS proximal-distal activation sequence. In order to reconstruct the chronology of the entire macroreentry circuit and to identify the mid-diastolic isthmus (MDI), a specific setting of the window of interest was used, as previously described [1]. Chronological activation analysis on the entire cycle $(230 \mathrm{~ms})$ indicated the MDI (the interface between the red and the purple colors) of typical atrial flutter, but more anterior. A decapolar catheter showed a typical counterclockwise activation along tricuspidal annulus and an enormous delay from low lateral right atrium-CS ostium, more than would be expected in case of a slow conduction (low lateral-proximal CS interval of $120 \mathrm{~ms}$.). A high-density mapping around isthmus and CS was performed (Figure 3). The re-analysis of the chronological activation clearly showed a circuit propagation around the CS ostium with a very slow conduction in the anterior zone enlightened by the tight color progression, and counterclockwise activation of the right atrium lateral wall (Figure 3). In anterior zone of CS ostium diastolic fragmented electrograms were detected. Entrainment that could have confirmed that suspected path was truly in the circuit, was not performed to avoid atrial fibrillation degeneration. After preventive His position localization, radiofrequency delivering (35 W) was effective to interrupt the arrhythmia in 3 sec. Energy delivering was continued to anchor the lesion to the inferior vena cava. Confirmation of successful ablation was determined by unsuccessful attempts at reinduction of the arrhythmia, in basal state and during infusion of isoproterenol.

\section{DISCUSSION}

In the era of transcatheter ablation procedures in the right and left atria to treat AF, incidence of organized macro-reentrant tachycardia, as a sequel of extensive linear ablation, is $5 \%-30 \%$ [2-4]. In Chugh et al. series on atrial flutters that arose after left atrial circumferential ablation of AF, in approximately $25 \%$ of cases they used the musculature of the CS [2]. Furthermore, Chae et al. reported a series of 21 micro-reentrant atrial tachycardias that occurred after circumferential pulmonary vein ablation. They indicated that the most common sites of origin

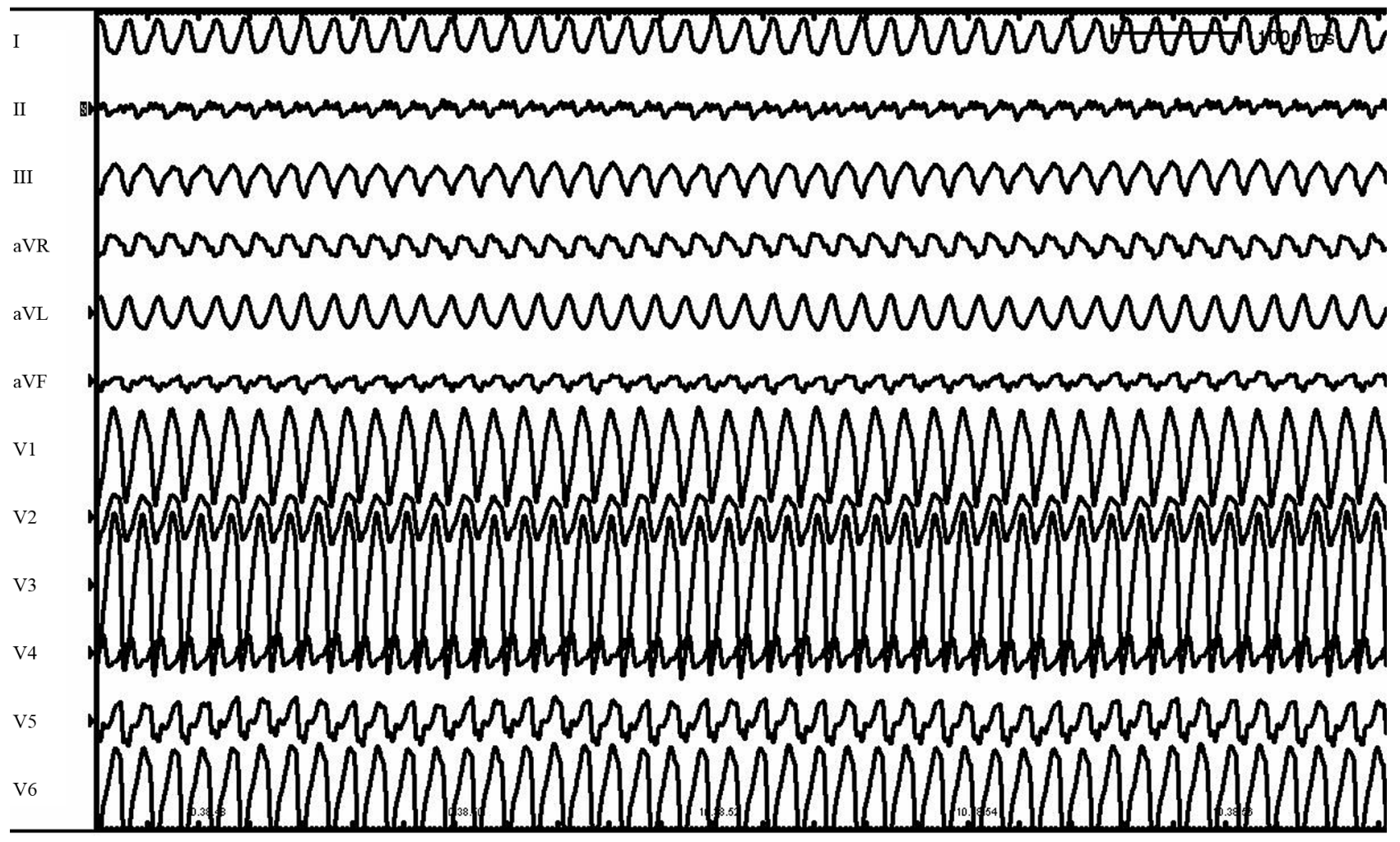

Figure 1. Induction of the huge very fast atrial tachycardia, $230 \mathrm{~ms}$ cycle length with $1 / 1$ atrio-ventricular conduction (ventricular rate of $260 \mathrm{bpm}$ ) and complete left bundle branch block. I, II, III, aVR, aVL, aVF, V1,V2, V3, V4, V5, V6 are surface electrograms. 


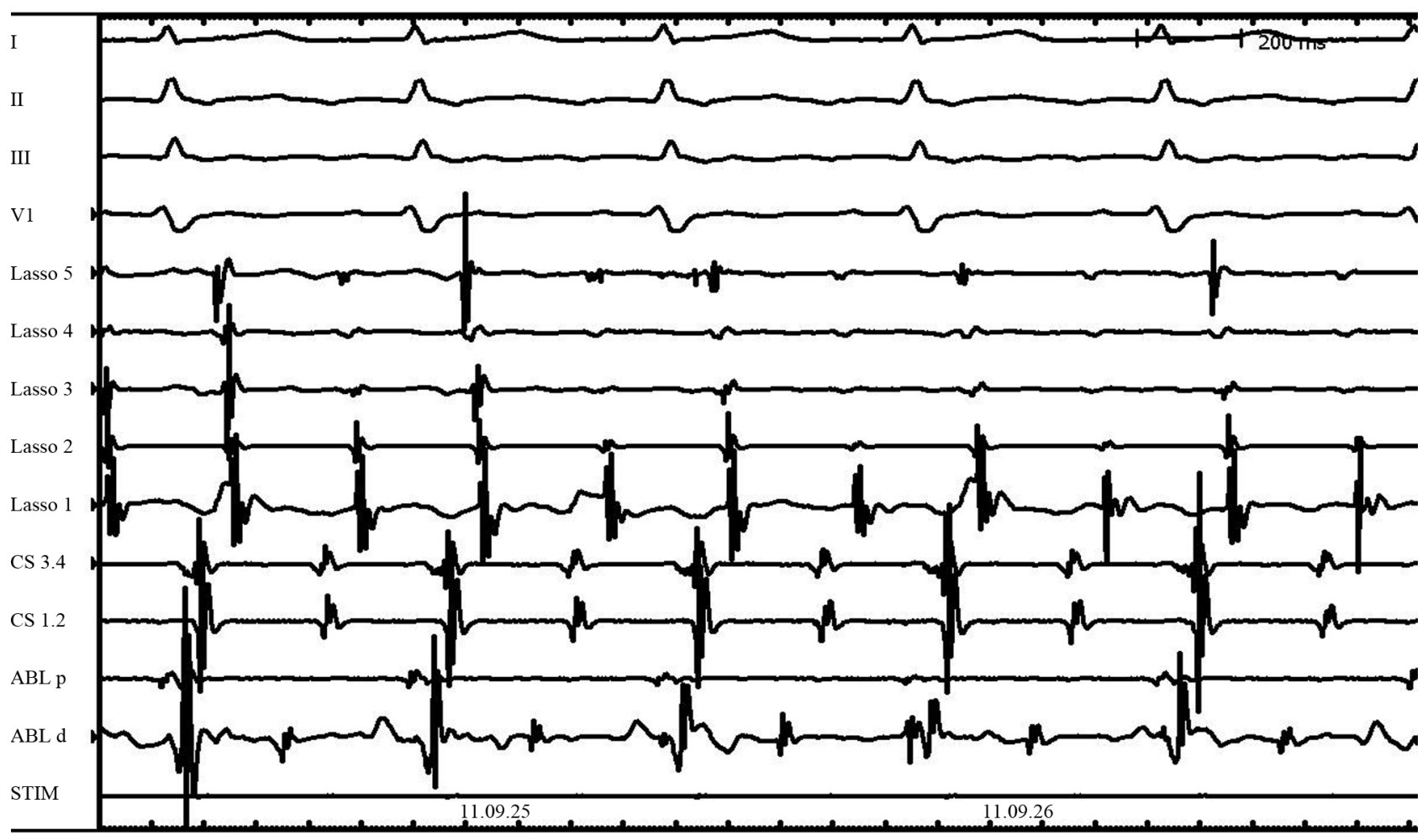

Figure 2. The atrial tachycardia with a 2/1 AV conduction without branch block. A decapolar catheter shows a typical counterclockwise activation along tricuspidal annulus and an enormous delay from low lateral right atrium-CS ostium (120 ms interval). I, II, III, V1 are surface electrograms; Lasso 5, Lasso 4, Lasso3, Lasso 2, Lasso 1 are electrograms from decapolar catheter; CS 3-4, CS 1-2 are electrograms from coronary sinus; ABL p, ABL d are proximal and distal electrograms from mapping/ablation catheter.

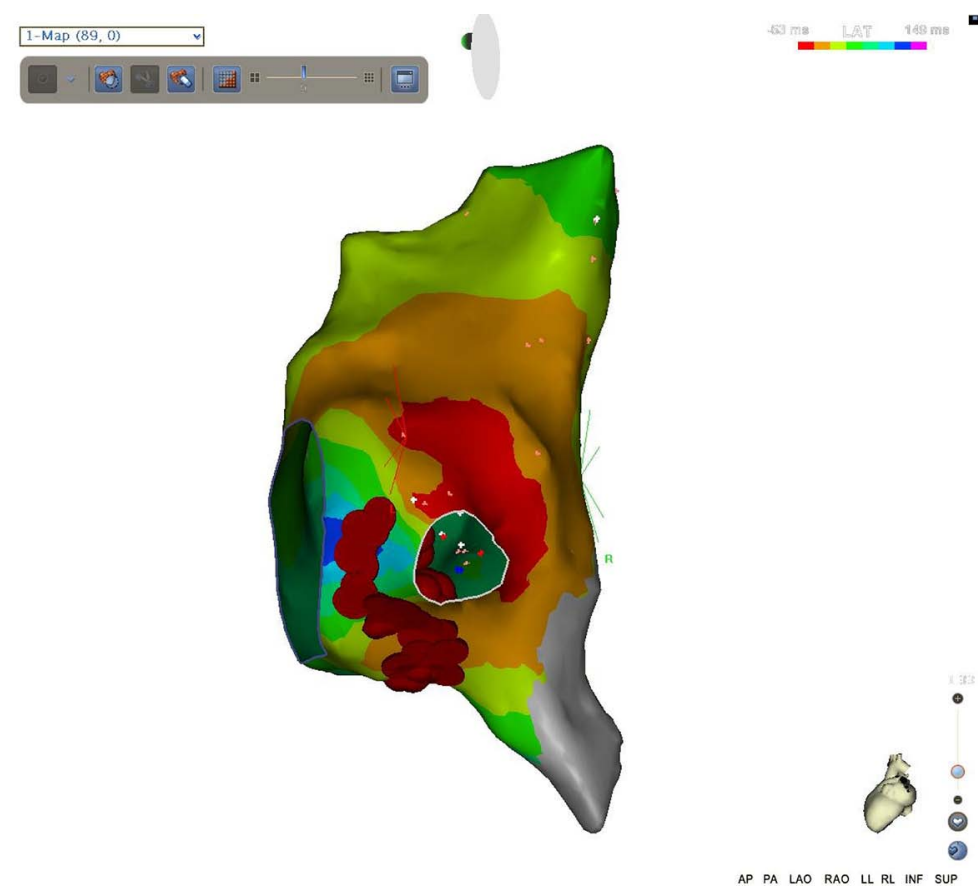

Figure 3. Isochronal map (LAO view) of tachycardia reentry counterclockwise circuit around coronary sinus ostium. Electrogram steps from $-53 \mathrm{~ms}$ to + 149 ms (about $90 \%$ of cycle length) are encoded with colors from red (earlier) to pink (latter). To note the slow conduction in the anterior zone enlightened by the tight color progression. 
were the CS, the antrium of a pulmonary vein, and the anterior wall of the left atrium or base of the left atrial appendage [3].

Morady et al. reported that $75 \%$ of atrial tachycardias occurred after wide-area radiofrequency ablation to isolate the pulmonary veins is caused by macroreentry also around CS [4].

Extensive liner lesions in right (isthmus, intercaval, superior vena cava) and left atria (circumferential pulmonary vein isolation, roof, anterior and posterior left isthmus) and normal anatomical barriers (tricuspidal annulus, Eustachian ridge and crista terminalis, scars) could contribute to the onset of conduction corridors: a manmade maze. In our case the stimulus goes slowly around CS ostium, especially in anterior zone, and spreads trough the right atrium corridors with a fast repetitive sequence with a counterclockwise activation of the lateral wall.

The result of extensive linear ablations is the onset of more organized tachyarrhythmias, often with rapid ventricular rates, persistent and difficult to suppress with rhythm control medications: from bad to worse.

\section{CONCLUSIONS}

In conclusion, our experience suggests that in case of extensive ablation lines for persistent atrial fibrillation or redo of paroxysmal atrial fibrillation, the highest therapeutic effectiveness is obtained by mapping and ablating all remaining circuits. At the last step, for the remaining circuit underlying a tachycardia with a more or less constant cycle length, electroanatomical mapping and ablation are mandatory.
This behaviour could prevent the arising of new tachyarrhythmias (man-made) which in the majority of cases is more symptomatic than atrial fibrillation.

\section{REFERENCES}

[1] De Ponti, R., Verlato, R., Bertaglia, E., Del Greco, M., Fusco, A., Bottoni, N., Drago, F., Sciarra, L., Ometto, R., Mantovan, R. and Salerno-Uriarte, J.A. (2007) Treatment of macro-re-entrant atrial tachycardia based on electroanatomic mapping: Identification and ablation of the middiastolic isthmus. Europace, 9, 449-457. http://dx.doi.org/10.1093/europace/eum055

[2] Chugh, A., Oral, H., Good, E., Han, J., Tamirisa, K., Lemola, K., Elmouchi, D., Tschopp, D., Reich, S., Igic, P., Bogun, F., Pelosi Jr., F. and Morady, F. (2005) Catheter ablation of atypical atrial flutter and atrial tachycardia within the coronary sinus after left atrial ablation for atrial fibrillation. Journal of the American College of Cardiology, 46, 83-91.

http://dx.doi.org/10.1016/j.jacc.2005.03.053

[3] Chae, S., Oral, H., Good, E., Dey, S., Wimmer, A., Crawford, T., Wells, D., Sarrazin, J.F., Chalfoun, N., Kuhne, M., Fortino, J., Huether, E., Lemerand, T., Pelosi, F., Bogun, F., Morady, F., Chugh, A., et al. (2007) Atrial tachycardia after circumferential pulmonary vein ablation of atrial fibrillation: Mechanistic insights, results of catheter ablation, and risk factors for recurrence. Journal of the American College of Cardiology, 50, 1781-1787. http://dx.doi.org/10.1016/j.jacc.2007.07.044

[4] Morady, F., Oral H. and Chugh, A. (2009) Diagnosis and ablation of atypical atrial tachycardia and flutter complicating atrial fibrillation ablation. Heart Rhythm, 6, S29S32. http://dx.doi.org/10.1016/j.hrthm.2009.02.011 